Bangladesh J. Sci. Res. 27(2): 187-200, 2014 (December)

\title{
EFFECTS OF VISCOUS DISSIPATION AND THERMAL RADIATION ON MHD FORCED CONVECTIVE HEAT AND MASS TRANSFER FLOW OF NON- NEWTONIAN POWER LAW FLUID WITH HEAT GENERATION
}

\author{
M. A. Samad* and C. Podder ${ }^{1}$ \\ Department of Applied Mathematics, Dhaka University, Dhaka-1000, Bangladesh
}

\begin{abstract}
An analysis is carried out to investigate the effects of forced convection heat and mass transfer of an electrically conducting, non-Newtonian power-law fluid past a stretching sheet. This has been done under the simultaneous action of suction, radiation, uniform transverse magnetic field, heat generation and viscous dissipation. The stretching sheet is assumed to continuously moving with a power-law velocity and maintaining a uniform surface heat flux. The governing nonlinear partial differential equations are transformed into a system of nonlinear ordinary differential equations using appropriate similarity transfor-mations. The resulting dimensionless equations are solved numerically using sixth order Runge-Kutta integration scheme with Nachtsheim-Swigert shooting iterative technique. A systematical study of numerical results for the non-dimensional velocity, temperature and concentration profiles are presented graphically. Due to physical and engineering interest, the viscous drag or local Skin-friction coefficient, heat transfer rate or local Nusselt number and mass transfer rate or local Sherwood number are represented in tabular form to illustrate the details of flow characteristics and their dependence on all physically important parameters of different non-Newtonian fluids. A comparison of the present study and previously published paper has also been shown in tabular form.
\end{abstract}

Key words: Non-Newtonian power-law fluid, stretching sheet, forced convec-tion, heat generation, thermal radiation, viscous dissipation.

\section{Introduction}

In the recent time, the problem of boundary-layer theory has spread all over the world, together with modern researches; it constitutes one of the most important pillars of fluid mechanics. The study of MHD forced convective flow of non-Newtonian power law fluid past a continuously moving electrically conducting stretching sheet in presence of thermal radiation, heat generation and viscous dissipation has received great attention of many scientists for its necessity in manufacturing and chemical processing system. The heat, mass and momentum transfer in the laminar boundary layer flow of non-Newtonian power law fluid on stretching sheets are important from a theoretical as well as practical point of view because of their wider applications to polymer technology, metallurgy, many mechanical forming processes, such as extrusion, melt-spinning, cooling, manufacture of plastic and rubber sheets, glass blowing, continuous casting and spinning

*Corresponding author: <masamad@gmail.com>. Department of Mathematics, Barisal University, Barisal, Bangladesh. 
of fibers etc. The interaction of radiation with hydromagnetic flow has become industrially more prominent in the processes wherever high temperatures occur. Nuclear power plants, gas turbines and the various propulsion devices for aircrafts, missiles, satellites and space vehicles are examples of such engineering areas. Forced convection should be considered as one of the main methods of useful heat transfer as significant amounts of heat energy can be transported very efficiently and this mechanism is found very commonly in everyday life, including central heating, air conditioning, steam turbines and in many other engineers designing or analyzing heat exchangers, pipe flow, and flow over a plate at a different temperature than the stream. Some literature surveys and reviews of pertinent work in this field are documented by Sakiadis (1961) first presented boundary layer flow over a continuous semi-infinite sheet moving with constant speed through a quiescent fluid environment. Elbashbeshy (1998) investigated heat transfer over a stretching surface with variable and uniform surface heat flux subject to injection and suction. Vajravelu and Hadjinicolaou (1997) studied the convective heat transfer in an electrically conducting fluid near an isothermal stretching sheet and they studied the effect of internal heat generation or absorption. Howell et al. (1997) analyzed momentum and heat transfer on a continuous moving surface in a power law fluid. Erickson et al. (1966) studied heat and mass transfer on a moving continuously moving surface. Rahman et al. (2008) investigated MHD forced convective flow of a micropolar fluid past a non-linear stretching sheet with a variable viscosity and Rahman et al. (2009) analyzed heat transfer in a micropolar fluid along a non-linear stretching sheet with a temperature-dependent viscosity and variable surface temperature. The magnetohydrodynamic boundary layer on a flat plate has been studied by Glauert (1961). The MHD boundary layer flow over a continuously moving plate for a micropolar fluid has been studied by Rahman and Sattar (2006) and Raptis (1998). Anderson et al. (1992) and Mahmoud and Mahmoud (2006) adopted the non-linearity relation as power-law dependency of shear stress on rate of strain. Dandapaat and Gupta (2005) extended the problem to study heat transfer and Datti et al. (2005) analyzed the problem over a non-isothermal stretching sheet. Cortell (2008) analyzed the effects of viscous dissipation and radiation on the thermal boundary layer over a nonlinearly stretching sheet.

Chen (2008) studied the effects of magnetic field and suction/injection on the flow of powerlaw non-Newtonian fluid over a power law stretched sheet subject to a surface heat flux. This problem studied by Chen (2008) has been extended to investigate the effects of thermal radiation, heat generation and viscous dissipation. Numerical and graphical computations for the velocity, temperature and concentration profiles have been carried out of different values of Suction parameter $\left(f_{w}\right)$, Prandtl number $(\operatorname{Pr})$, Magnetic parameter $(M)$, Radiation parameter $(N)$, Heat source parameter $(Q)$, Schmidt number $(S c)$, Eckert number $(E c)$, velocity index $(p)$ and powerlaw fluid index $(n)$. The local skin friction coefficient, local Nusselt number and local Sherwood number have also been obtained to investigate more practical and physical effect of above all parameters on different non-Newtonian fluids. A comparison of the present study with Chen (2008) has been done and given in tabular form (Table 1). 
Table 1. Comparison of skin friction coefficient $\mathbb{Z}\left(\mathrm{C}_{-\mathrm{f}}^{-}\right)$and local Nusselt number $\left(\mathrm{Nu} u_{x}\right)$.

\begin{tabular}{|c|c|c|c|c|c|}
\hline \multicolumn{2}{|c|}{$\operatorname{Pr}=5.0, p=0.5, n=1.0$} & \multicolumn{2}{|c|}{$R e_{x}^{\frac{1}{\ln +1}} \mathrm{C}_{\mathrm{f}}$} & \multicolumn{2}{|c|}{$R e_{x}^{\frac{-1}{(n+1)}} N u_{x}$} \\
\hline$M$ & $f_{w}$ & Chen (2008) & Present study & Chen (2008) & Present study \\
\hline 00 & $\tilde{\mathrm{N}} 0.2$ & $\tilde{\mathrm{N}} 1.345021$ & $\underset{\tilde{N} 1}{\tilde{N}} .3453779$ & 1157.198 & 1157.9345 \\
\hline 0.0 & 0.0 & $\tilde{N} 1.540734$ & Ñ̃ .5412588 & 1.694009 & 1.6941463 \\
\hline 0.0 & 0.6 & $\tilde{N}$ D.270891 & $\tilde{N}$ D.2718551 & 3.920419 & 3.9209955 \\
\hline $1 \cap$ & $\tilde{\mathrm{N}} \cap ?$. & $\tilde{N} \cdot 373803$ & $\tilde{\mathrm{N}} 732.38165$ & 1010724 & 10107078 \\
\hline 1.0 & 0.0 & $\tilde{N} D .519363$ & $\tilde{\mathrm{N}} 2.5193792$ & 1.578428 & 1.5785832 \\
\hline 1.0 & 0.6 & $\tilde{N} B .198565$ & $\tilde{\mathrm{N}} 3.1985873$ & 3.866662 & 3.8672219 \\
\hline 5.0 & Ñ 0.2 & Ñ4.529439 & -4.5294577 & 0.692476 & 0.6927982 \\
\hline 50 & 00 & -477.67 .10 & $\tilde{\mathbf{N}} 4.7262300$ & 133,0497 & 137.15455 \\
\hline 5.0 & 0.6 & $\tilde{\mathrm{N}} 5.366930$ & Ñ 5.3669466 & 3.754876 & 3.7552709 \\
\hline
\end{tabular}

\section{Governing equations of motion}

Let us consider a steady two dimensional MHD forced convective laminar boundary layer flow of a viscous incompressible and electrically conducting fluid obeying the power-law model along a permeable stretching sheet with the influence of thermal radiation, heat generation and viscous dissipation. The origin is located at the slit through which the sheet is drowning through the fluid medium. The flow is assumed to be in the $x$-direction, which is taken along the sheet and $y$-axis is normal to it. Two equal and opposite forces are introduced along the $x$-axis, so that the sheet is stretched keeping the origin fixed (Fig. 1). The plate is maintained at a constant temperature $T_{w}$ and the ambient temperature is $T_{\infty}$ This continuous sheet is assumed to move with a velocity according to a power-law form, i.e. $u_{w}=C x^{P}$. The fluid is considered to be gray, absorbing emitting radiation but non-scattering medium and the Rosseland approximation is used to describe the radiative heat flux in the energy equation. The radiative heat flux in the $x$-direction is considered negligible in comparison to the $y$-direction. A strong magnetic field $B$ is applied in $y$-direction. The magnetic Reynolds number is assumed to be small so that induced magnetic field is negligible. The electrical current flowing in the fluid gives rise to an induced magnetic field if the fluid were an electrical insulator, but here have taken the fluid to be the electrically conducting. Hence, only the applied magnetic field $B$ Plays a role which gives rise to magnetic

forces $F_{x}=\frac{\sigma B u}{\rho}$ in the $x$-direction, where $\sigma$ is electrical conductivity, $B$ is the uniform magnetic field strength (magnetic induction) and $\rho$ is the density of the fluid. 


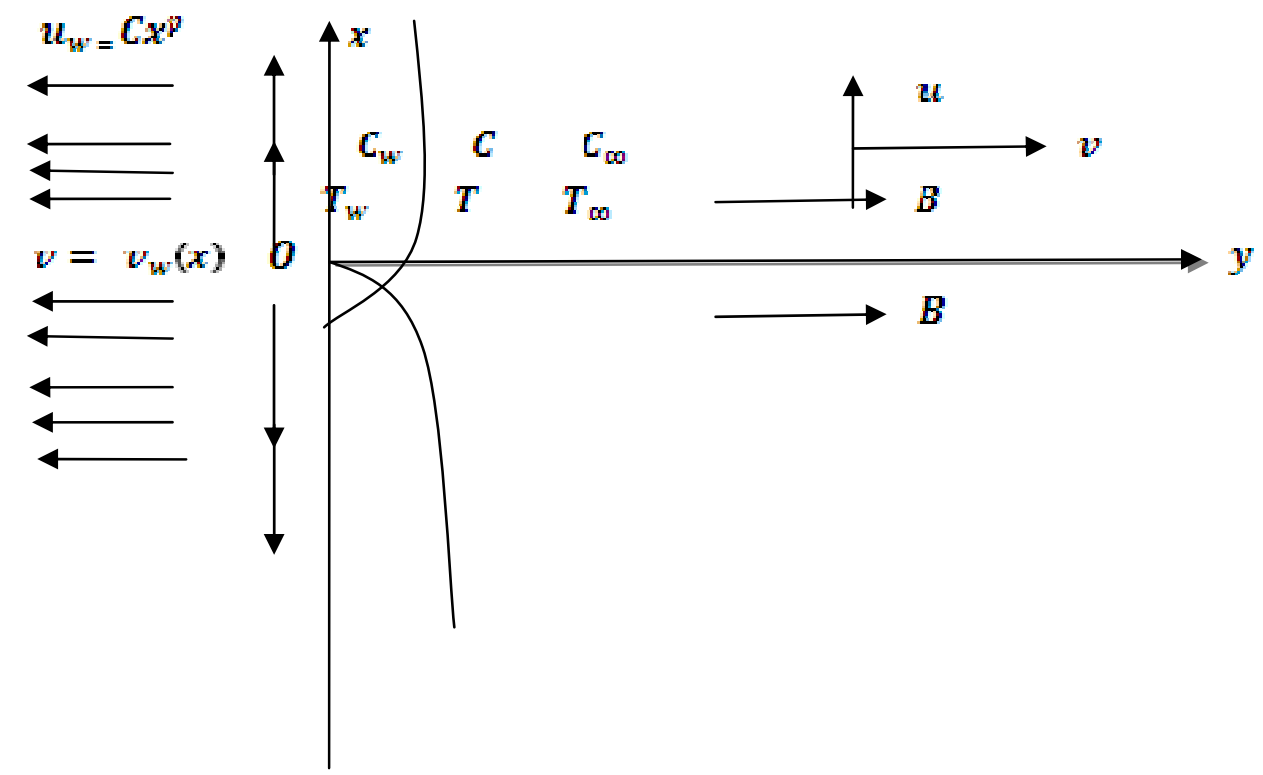

Fig. 1. The physical model of boundary lay.

Under the usual boundary layer approximation, the flow and heat transfer in the presence of thermal radiation, heat generation and viscous dissipation are governed by the following equations:

Continuity equation: $\frac{\partial u}{\partial x}+\frac{\partial v}{\partial y}=0$

Momentum equation: $u \frac{\partial u}{\partial x}+v \frac{\partial u}{\partial y}=\frac{K}{\rho} \frac{\partial}{\partial y}\left(\left|\frac{\partial u}{\partial y}\right|^{n-1} \frac{\partial u}{\partial y}\right)-\frac{\sigma B^{2} u}{\rho}$

Energy equation:

$$
u \frac{\partial T}{\partial x}+v \frac{\partial T}{\partial y}=\alpha \frac{\partial^{2} T}{\partial y^{2}}+\frac{Q_{0}}{\rho C_{p}}\left(T-T_{w}\right)-\frac{1}{\rho C_{p}} \frac{\partial q_{r}}{\partial y}+\frac{K}{\rho C_{p}}\left(\left|\frac{\partial u}{\partial y}\right|^{n-1} \frac{\partial u}{\partial y}\right)^{2}
$$

Concentration equation: ${ }^{u} \frac{\partial C}{\partial x}+v^{\partial C}=D_{m}{ }^{\partial^{2} C}$

where $u$ and $v$ are the velocity components along $x$ and $y$-directions respectively, $T$ be the temperature of the fluid layer, $v$ is the kinematic viscosity, $p$ is the density, $\sigma$ is the electric conductivity, $\alpha$ is the thermal conductivity, $B$ is the uniform magnetic field, $C_{p}$ is the specific heat at constant pressure, $\alpha$ is the thermal diffusivity, $q_{r}$ is the radiative heat flux, $K$ is the consistency coefficient, $n$ is the flow behavior/power-law fluid index. The radiative heat flux $q r$ is described by the Rosseland approximation such that, 


$$
q_{r}=-\frac{4 \sigma_{1}}{3 k_{1}} \frac{\partial T^{\mathbf{4}}}{\partial y}
$$

where $\sigma_{1}$ is the Stefan-Boltzman constant and $k_{1}$ is the Rosselend mean absorption coefficient. It is assumed that the temperature difference within the flow are sufficiently small such that $T^{4}$ in a Taylor series about the free steam temperature $T_{\infty}$ and then neglecting higher-order terms. This results in the following approximation:

$$
T^{4} \times 4 T_{\infty}^{3}-3 T_{\infty}^{4}
$$

Using (5) and (6) in $\frac{\partial q_{r}}{\partial y}$ which appear in equation (4), we have,

$$
\frac{d q r}{\partial y}=-\frac{16 \sigma_{1} \gamma_{x}^{2}}{3 k_{1}} \frac{\partial y^{\prime}}{\partial y^{2}}
$$

Introducing $\frac{\partial q_{r}}{\partial y}$ in (3), we obtain the following governing boundary layer equations:

Continuity equation: $\frac{\partial u}{\partial x}+\frac{\partial v}{\partial y}=0$

Momentum equation: $u \frac{\partial u}{\partial x}+v \frac{\partial u}{\partial y}=\frac{K}{\rho} \frac{\partial}{\partial y}\left(\left|\frac{\partial u}{\partial y}\right|^{n-1} \frac{\partial u}{\partial y}\right)-\frac{\sigma B^{2} u}{\rho}$

Energy equation:

$$
\begin{aligned}
& u \frac{\partial l}{\partial x}+v \frac{\partial l}{\partial y}=\alpha \frac{\partial^{2} l}{\partial y^{2}}+\frac{\varphi_{0}}{\rho C_{p}}\left(\mathrm{~T}-\mathrm{T}_{x}\right)+\frac{1}{\rho C_{p}} \frac{16 \sigma_{1} l_{w}^{3}}{3 k_{1}} \frac{\partial^{2} l}{\partial y^{2}}+\frac{K}{\rho C_{p}}\left(\left|\frac{\partial u}{\partial y}\right|^{n-1} \frac{\partial u}{\partial y}\right)^{2} \\
& \text { Concentration equation: } u \frac{\partial C}{\partial x}+v \frac{\partial C}{\partial y}=n_{m} \frac{\partial^{2} C}{\partial y^{2}}
\end{aligned}
$$

The appropriate boundary conditions are:

$$
\left.\begin{array}{rccc}
u_{w}=C x^{p}, & v=v_{w}, \quad \frac{\partial T}{\partial y}=-\frac{q_{w}}{\kappa}, & C=C_{\infty}+b x & \text { at } y=0, \quad x>0 \\
u_{w} \rightarrow 0, & T \rightarrow T_{\infty}, \quad C \rightarrow C_{\infty} & \text { as } y \rightarrow \infty
\end{array}\right\}
$$

where $v_{w}$ is the surface mass flux and $q_{w}$ is the surface heat flux. It should be noted that positive $p$ indicates that the surface is accelerated while negative $p$ implies that surface is decelerated from the slit. Positive $v_{w}$ is for fluid injection and negative $v_{w}$ for fluid suction at the sheet surface. In order to obtain a similarity solution of the problem, we introduce a similarity parameter $\delta(x)$, such that $\delta(x)$ is a length scale. Introducing the following dimensionless quantities, we have 
$\eta=\frac{y}{\partial(x)}=\left(\frac{C^{2-n}}{\frac{F}{\rho}}\right)^{1 /(n+1)} x^{\frac{(p(2-n)-1)}{n+1} y}$

$\psi=\left(\frac{c^{1-2 n}}{K / \rho}\right)^{-1 / n+1)} x^{\frac{\phi(n-1)+1}{n+1}} f(\eta)$

$\theta(\eta)=\frac{\left(I-Y_{\infty}\right) H e_{x}^{\frac{1}{n 11}}}{q_{w}^{x} / k}$

$\phi(\eta)=\frac{C-C_{0 i}}{b x}$

where $\psi$ is the stream function, $\eta$ is the dimensionless distance normal to the sheet, $f$ is the dimensionless stream function and $\theta$ and $\phi$ is the dimensionless fluid temperature and concentration respectively.

The stream function $\psi$ satisfy the continuity equation (1), so we get the velocity components $u$ and $v$ as follows.

$u=\frac{\partial \psi}{\partial y}=u_{w} f^{\prime}(\eta)$

$v=-\frac{\partial \psi}{\partial x}=-u_{w} R e_{x}^{-\frac{1}{(n+1)}}\left[\frac{\varphi(2 n-1)+1\}}{(n+1)} f(\eta)+\frac{\{(2-n)-1\} \eta}{(n+1)} f^{s}(\eta)\right]$

Introducing similarity variables from equations (13) - (16) and using the transformations we get the following:

Momentum equation:

$\left(\left|f^{s}\right|^{n-1} f^{r}\right)^{r}+\frac{[p(2 n-1)+1\}}{(n+1)} f f^{\prime s}-p\left(f^{\prime}\right)^{2}-M f^{\prime}=0$

Energy equation:

$\frac{3 N+4}{3 N \text { Pr }} \theta^{\prime e}+\frac{6(2-n)-1\}}{(n+1)} f^{\prime \prime} \theta+\frac{6(2 n-1)-1\}}{(n+1)} j \theta^{\prime}+v \theta+E c\left(\left|f^{\prime \prime r}\right|^{n-1} f^{\prime \prime}\right)^{2}=0$

Concentration equation:

$$
\frac{1}{s c} \phi^{s}+\frac{[(2 n-1)+1]}{(n+1)} f \phi^{s}-f^{r} \phi=0
$$


The transformed boundary conditions are:

$$
\begin{aligned}
& \left.\begin{array}{rr}
f^{s}=1, \quad f=\frac{n+1}{p(2 n-1)+1} f_{W}, \theta^{4}=-1, & \phi=1 \text { at } \eta=0 \\
f^{s} \rightarrow 0, \quad \theta \rightarrow 0, \quad \phi \rightarrow 0 & \text { as } \eta \rightarrow \infty
\end{array}\right\} \\
& f_{w}=-\frac{n_{w}}{u_{w}} R e_{w}^{\frac{1}{(n+1)}} \text { is the suction parameter, } M=\begin{array}{l}
\sigma B^{2} x \\
\rho u_{w}
\end{array} \text { is the magnetic field }
\end{aligned}
$$
generalized Prandtl number, $N=\frac{\kappa \kappa_{1}}{4 \sigma_{1} T_{\infty}^{a}}$ is the radiation parameter, $Q=\frac{Q_{0} X}{u_{w} p C_{p}}$ is the heat source param

$$
E c=\frac{\left(u_{w}^{\frac{n+1}{2}}\right)^{2}}{C_{p} \cdot \frac{q_{w}}{k} x^{n}} R e_{x}^{\frac{n}{n+1}} \text { is the Eckert number, } S c=\frac{u_{w} x^{2}}{D_{m}} R e_{x}^{\frac{-2}{(n+1)}} \text { is the }
$$

local Schmidt number. Here, we note that the magnetic field strength $B$ should be proportional to $x$ to the power of $\frac{p-1}{2}$ to eliminate the dependence of $M$ on $x$, i.e. $B(x)=B_{0} x^{6-11 / 2}$, where $B_{0}$ is a constant.

\section{Results and Discussion}

In order to analyze the results, graphical computations have been carried out for the different values of Suction parameter $\left(f_{w}\right)$, Prandtl number $(\operatorname{Pr})$, Magnetic parameter $(M)$, Radiation parameter $(N)$, Heat source parameter $(Q)$, Schmidt number $(S C)$, Eckert number $(E c)$, velocity index $(b)$ and power-law fluid index $(n)$.

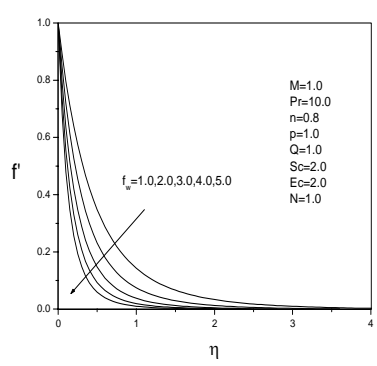

Fig. 2(a). Suction parameter (iw) effect on velocity profiles.

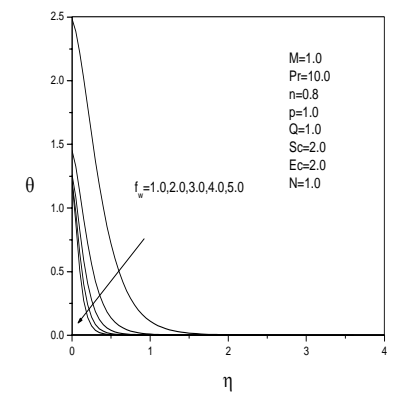

Fig. 2(b). Suction parameter

(iw) effect on temperature profiles.

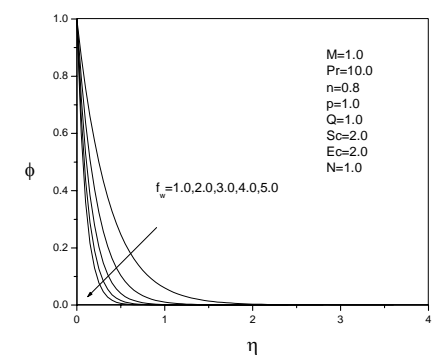

Fig. 2(c). Suction parameter $(f w)$ effect on concentration profiles. 
In Figs. 2a, b, c the dimensionless velocity, temperature and concentration profiles are plotted, respectively showing the effect of suction parameter $\left(f_{w}\right)$. Due to suction some of the retarded fluid particles are taken out from the boundary layer and thus prevent the boundary layer separation. In Fig. 2(a) the velocity decreases with the increase of suction parameter. This can be explained by the fact that when the suction parameter $\left(f_{w}\right)$ increases, some matter is removed from the system and the velocity gets retarded most rapidly. Also the momentum boundary layer thickness decreases with the increase of the suction parameter $\left(f_{w^{*}}\right)$ and thus reduces the chance of the boundary layer to the transition to turbulence. From Figs. 2b,c there is similarity for temperature and concentration profiles that, the temperature and concentration of the fluid particle are low for a fluid of higher suction. Thus it reduces the thermal and concentration boundary layer thickness with the increase of the suction parameter $\left(f_{1} w\right)$ to a significant amount. The reduction in the velocity, temperature and concentration profiles also indicates that suction stabilizes the velocity, temperature and concentration boundary layer. So, suction can be used very effectively in controlling the flow field.

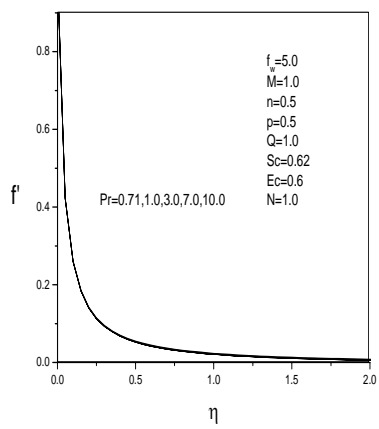

Fig. 3 (a). Prandtl number (Pr) effect on velocity profiles.
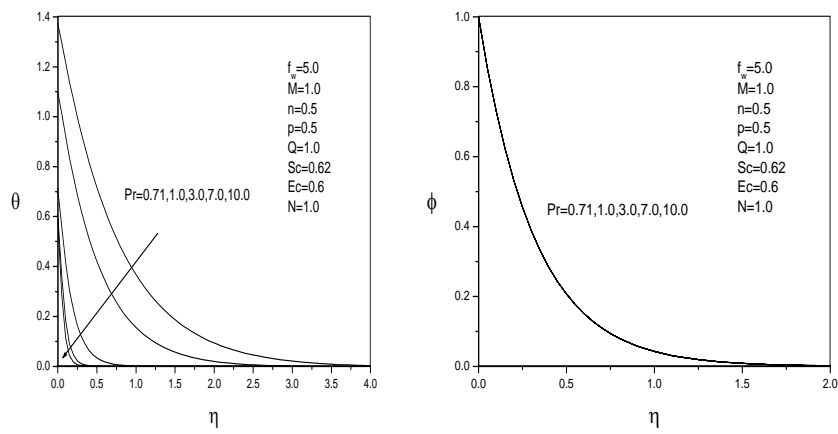

Fig. 3 (b). Prandtl number (Pr) Fig. 3 (c). Prandtl number (Pr) effect on temperature effect on concentration profiles. Profiles.

In the forced convection, the velocity and concentration are generally high in comparison with the effect of Prandtl number. So, the momentum and concentration boundary layer thickness remain fixed with the increase of the Prandtl numberPr. From Figs 3a, $c$ it is observed that there is no effect of Prandtl number PrI on velocity and concentration profiles. The velocity and concentration of the stretching sheet and the fluid particle remain same with the increase of Prandtl number PrI. From Fig. 3(b), the temperature decrease with the increase of the Prandtl number $\mathrm{Pr}$. This is because of lower Prandtl number $\mathrm{Pr}$, the heat transfer at much slower rate. On the other hand for higher Prandtl numberPr the heat transfer at much higher rate. The thermal boundary layer thickness decreases to a large extent with the increase of the Prandtl 
number $\mathrm{Pr}_{\mathbf{m}}$. However, for $\boldsymbol{P}_{\mathbf{r}}=0.71, \mathbf{1 . 0}$ wall temperature is very high compared to larger values. It follows that $\mathrm{Pr}$ strongly influences the relative growth of the thermal boundary layer.
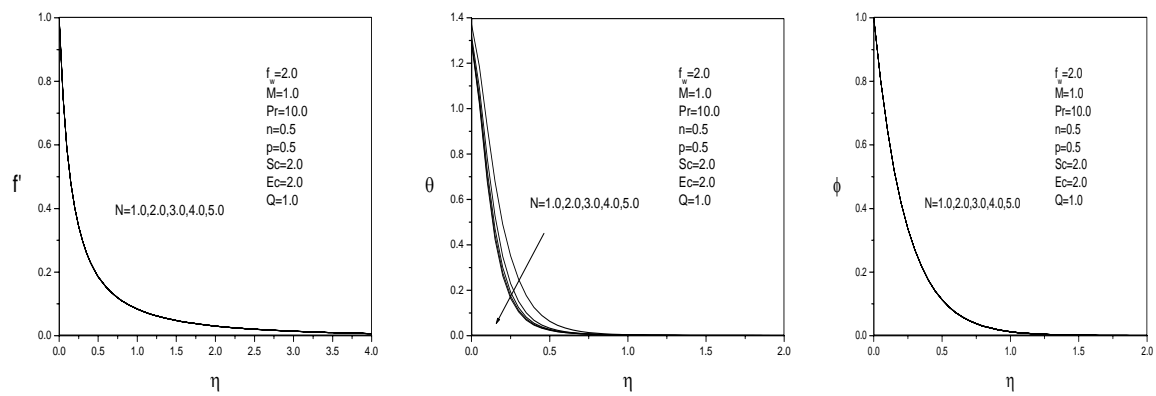

Fig. 4(a). Radiation parameter $(N)$ Fig. 4(b). Radiation parameter $(N)$ Fig. 4(c). Radiation parameter
effect on velocity profiles.
effect on temperature
profiles.
$(N)$ effect on concentration profiles.

From Figs 4a, c it is seen that the velocity and concentration remain unchanged with the increase of the radiation parameter $(N)$. The momentum and concentration boundary layer thickness remain unaffected with the increase of the radiation parameter $(N)$. So the velocity and concentration of the stretching sheet and the fluid particle remain same with the increase of radiation parameter $(N)$. Thus there is no visible effect on velocity and concentration profiles. Again, from Fig. 4(b) the temperature profiles decrease as the radiation parameter $(N)$ is increased. This can be explained by the fact that the thermal boundary layer thickness reduces very rapidly. For higher radiation the temperature decreases more quickly.

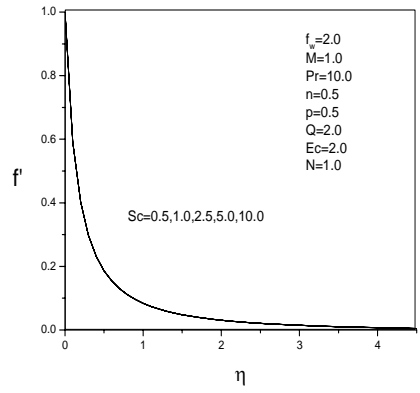

Fig. 5(a). Schimidt number (Sc) effect on velocity profiles
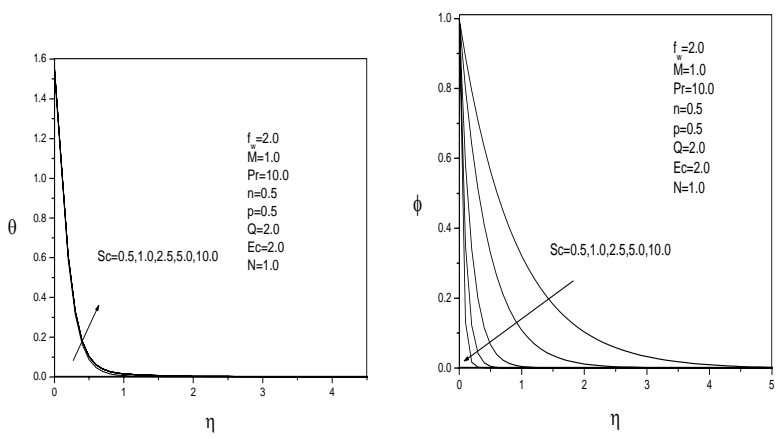

Fig. 5(b). Schimidt number (Sc) effect on temperature profiles
Fig. 5(c). Schimidt number $(S c)$ effect on concentration profiles 
From Figs 5a, $\mathrm{b}$ the momentum boundary layer remains unaffected and the temperature fields slightly increase with the increase of the Schmidt number $(5 \mathrm{c})$. It is seen from Fig. 5(c) that the concentration profiles rapidly decrease with the increase of Schmidt number $(5 \mathrm{c})$. It indicates that Schmidt number $(50)$ can be used very effectively to control the concentration of the flow field.

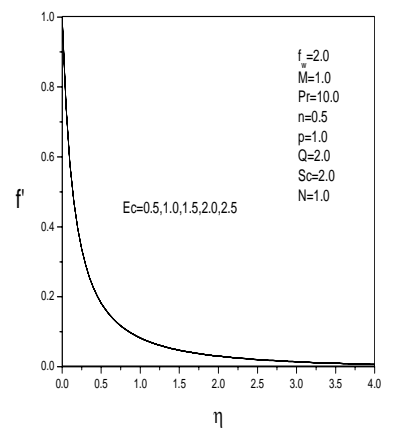

Fig. 6(a). Eckert number $(E c)$ effect on velocity profiles.

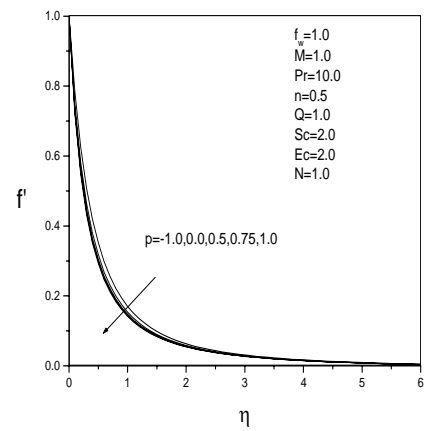

Fig. 7(a). Velocity index $(p)$ effect on velocity profiles $(n=0.5)$.

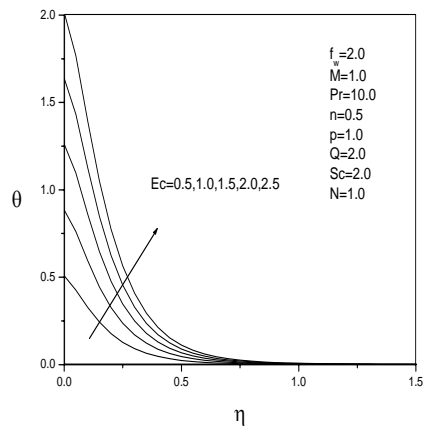

Fig. 6(b). Eckert number $(E c)$ effect on temperature profiles.

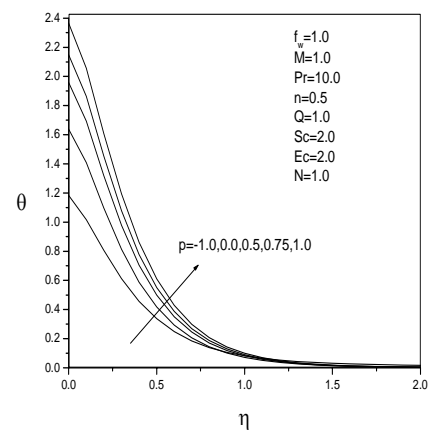

Fig. 7(b). Velocity index (p ) effect on emperature profiles $(n=0.5)$.

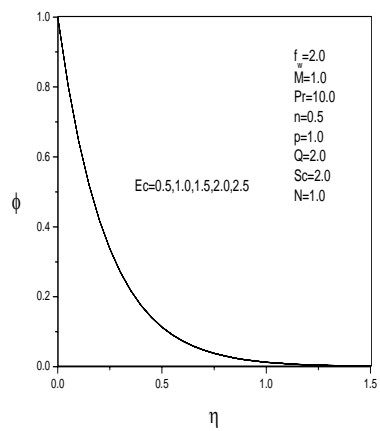

Fig. 6(c). Eckert number $(E c)$ effect on concentration profiles.

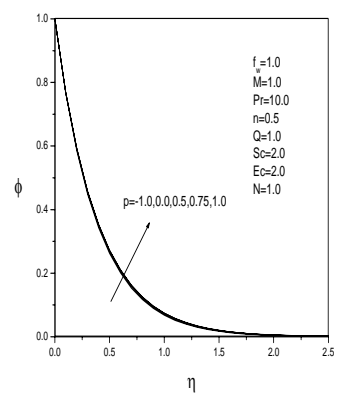

Fig. 7(c). Velocity index $(p)$ effect on concentration profiles $(n=0.5)$

From Figs 6a, c the momentum and concentration boundary layer thickness remain unaffected with the increase of the Eckert number $(E C)$. The velocity and concentration remain unchanged for the increase of the Eckert number $(E C)$. So, there is no significant visible effect on velocity and concentration profiles for Eckert number (EC). In Fig. 6(b) the temperature profiles rapidly increase with the increase of Eckert number $(E C)$. It indicates that viscous dissipation effect can be used very effectively to control the temperature of the flow field. 
Figs. 7a, 8a, 7b, 8b and Fig. 7c and 8c reveal the effect of velocity index $(p)$ for $(n<1)$ and $(n>1)$ on velocity, temperature and concentration profiles respectively. So, it can be concluded that the velocity profiles decrease for both pseudo-plastic and dilatants fluids. But the temperature profiles in Fig. $7 \mathrm{~b}$ and Fig. $8 \mathrm{~b}$ show that the temperature increase with the increase of velocity index $(p)$ for $(n<1)$ and the temperature decreases with the increase of velocity index $(p)$ for $(n>1)$. So, the pseudo-plastic and dilatants fluids show completely opposite behavior for temperature and concentration profiles. Also, we see that in case of pseudo-plastic fluid there is an increasing effect and for dilatants fluid there is a very significant decreasing effect on concentration profiles.
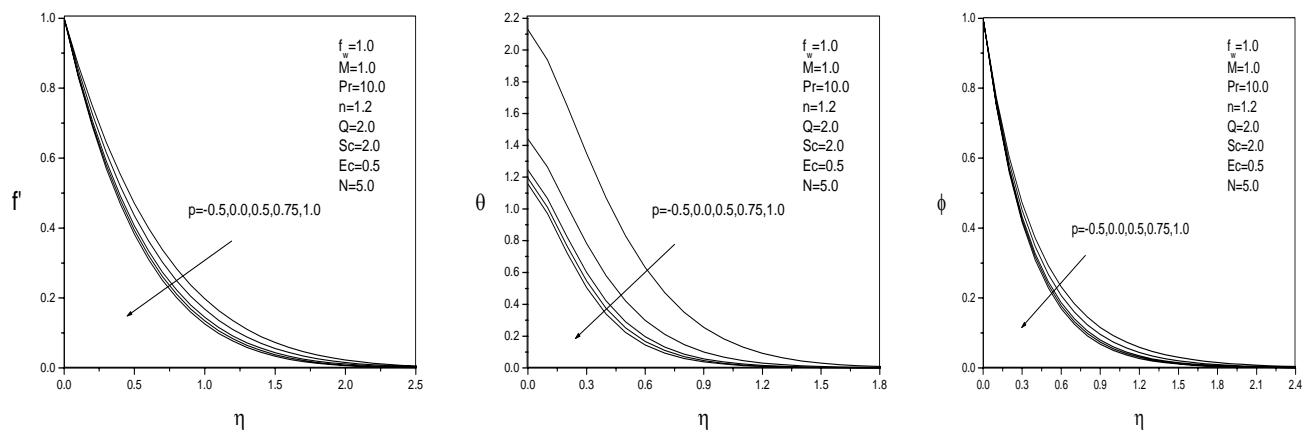

Fig. 8(a).Velocity index ( $p$ ) effect on Fig. 8(b). Velocity index ( $p$ ) on Fig. 8(c). Velocity index ( $p$ ) effect velocity profiles $(n=1.2)$.

effect temperature profiles $(n=1.2)$

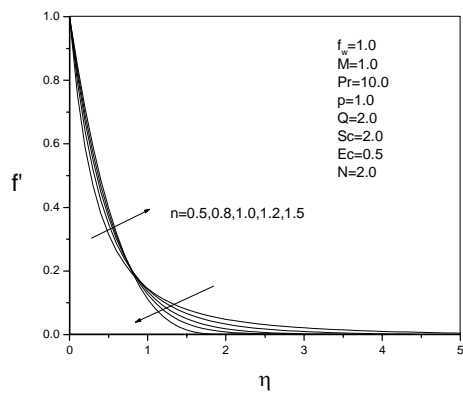

Fig. 9(a). Power-law index († ) effect on velocity profiles for accelerated profiles.

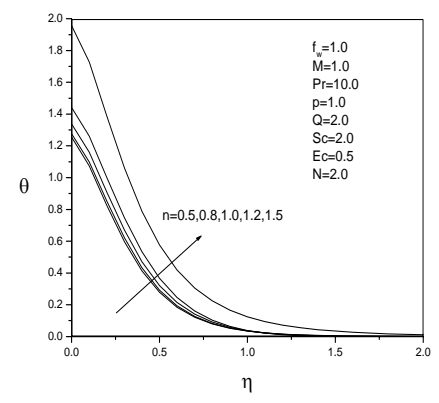

Fig. 9(b). Power-law index ( $"$ ) effect Fig. 9(c). Power-law index ( $n$ ) on temperature profiles for accelerated flow $(p=1,0)$. on concentration profiles $(n=1,2)$.

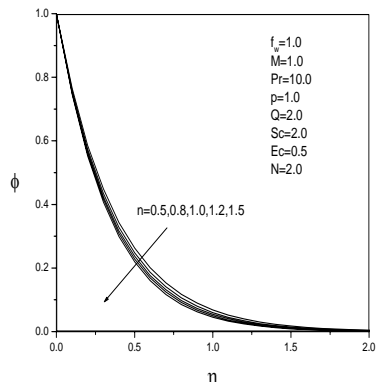
effect on concentration profiles for accelerated flow $(\nu=1.0)$.

The effect of the velocity, temperature and concentration fields due to power-law fluid index $(n)$ for accelerated flow $(p=1.0)$ and decelerated flow $(p=-0.5)$ are investigated in Figs. 9a, 9c, 10a, 10c, respectively. From Fig. 9a and Fig. 10a, it is seen that with the increase of 
power-law fluid index $(n)$, firstly the velocity increase then start to decrease, so we get a cross flow for both cases near $\eta=1.0$. For accelerated flow $(p=\mathbf{1 . 0})$ cross flow is shown near $\eta \ll 1.0$ and for decelerated flow cross flow is shown $\eta \gg 1.0$. Here, from Figs $9 \mathrm{~b}$ and $10 \mathrm{~b}$, there are same effects for velocity and temperature profiles for accelerated and decelerated flows. The temperature profiles increase with the increase of the power-law fluid index $(n)$ for both accelerated and decelerated flows. But there is a small variation of different fluids for accelerated flow $(p=1.0)$ and a large variation can be found among different fluids for decelerated flow $(p=-0.5)$. From Figs $9 \mathrm{c}$ and $10 \mathrm{c}$, we observe that the concentration profiles sharply decrease for accelerated flow $(p=1.0)$ and increase for decelerated flow $(p=-0.5)$ with the increase of power-law fluid index $(n)$ ). There is an opposite behavior in the concentration field due to accelerated and decelerated flows.
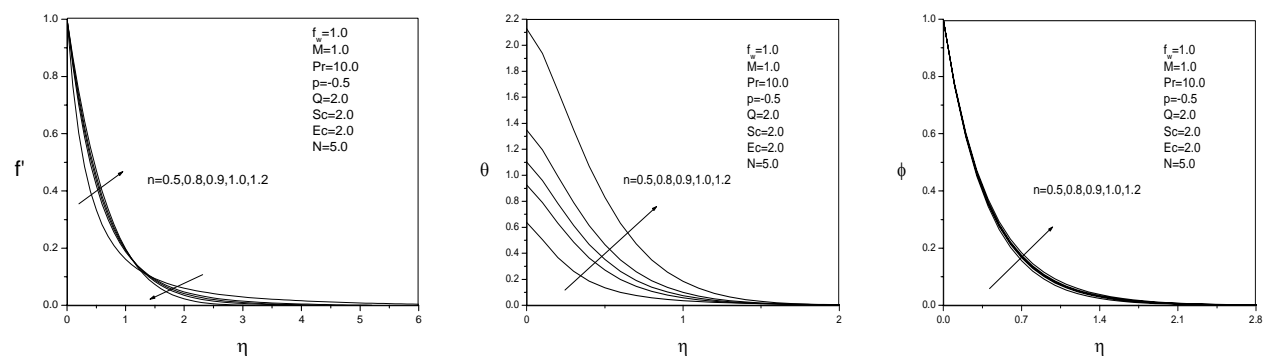

Fig. 10 (a). Power-law index ( $n$ ) effect Fig. 10 (b). Power-law index $(n)$ ) Fig. 10 (c). Power-law index $(n)$ on velocity profiles for decelerated flow $(p=-0.5)$. effect on temperature profiles for decelerated flow $(\eta=-0.5)$. effect on concentration profiles for decelerated flow $(\eta=-0 . \overline{5})$.

\section{Skin friction coefficient $\left(C_{f}\right)$, local Nusselt number $\left(N u_{x}\right)$ and local sherwood} number ( $S h$ )

The wall shear stress,

$$
\tau_{w}=\left.K\left(\left|\frac{\partial u}{\partial y}\right|^{n-1} \frac{\partial u}{\partial y}\right)\right|_{y=0}=\rho u_{w}^{2} R e_{x}^{-\frac{1}{(n+1)}}\left|f^{n}(0)\right|^{n-1} f^{s x}(0)
$$

Skin friction coefficient

$$
\mathrm{C}_{\mathrm{f}}=\frac{\tau_{w}}{\frac{1}{2} \rho u_{W}^{2}} \text { or, } \quad R e_{w}^{\frac{1}{(n+1)}} \mathrm{C}_{\mathrm{f}}=2\left|f^{\prime \prime}(0)\right|^{n-1} f^{x}(0)
$$

The local Nusselt number, $N u_{x}=\frac{h x}{k}=\frac{R e^{1 /(n+1)}}{\theta(0)}$ or, $R e_{x}^{\frac{-1}{(n+1)}} N u_{x}=\frac{1}{\theta(0)}$ and

local Sherwood number, $S h=\frac{x M_{W}}{D_{m}\left(C-C_{m}\right)}$ or, $S h R e_{x}^{\frac{-1}{(n+1)}}=-\phi^{x}(0)$. 
Here, skin friction coefficient $\left(C_{f}\right)$, local Nusselt number $\left(N u_{x}\right)$ and local Sherwood number (Sh) are proportional to $2\left|f^{t}(0)\right|^{n-1} f^{s}(0), 1 / \theta(0)$ and $-\phi^{5}(0)$ respectively. The effects of respective parameters on skin friction coefficient $\left(C_{f}\right)$, local Nusselt number $\left(N u_{s}\right)$ and local Sherwood number $(S h)$ are shown in graphical form.

Graphical representration of skin friction coefficient, local Nusselt number and local Sherwood number
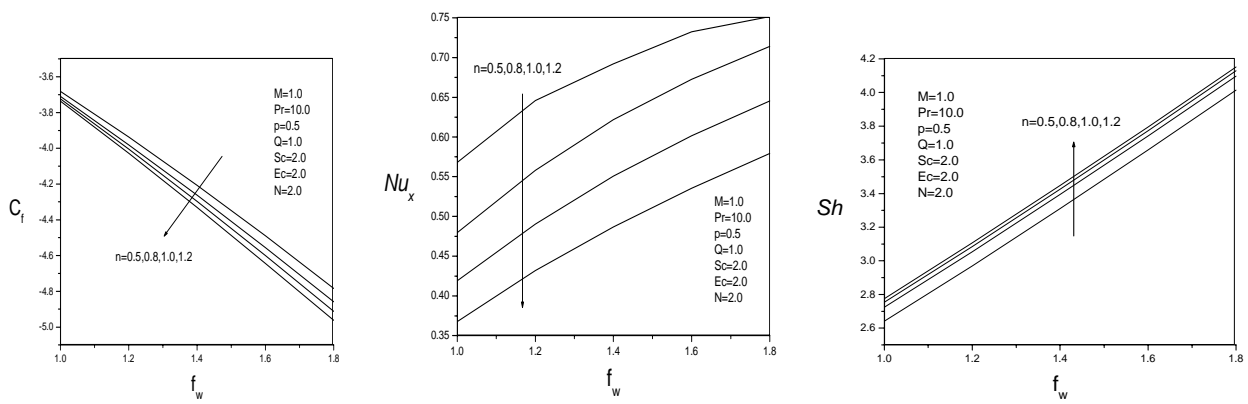

Fig. 11. Variation of skin friction coefficient $\left(C_{f}\right)$, local Nusselt number $\left(N u_{x}\right)$ and local Sherwood number $(5 h)$ as a function of $\left(f_{w}\right)$ at selected values of $n$.

\section{Conclusion}

From the above investigations, we can draw the following conclusions:

1. Suction has significant effects on the flow field and can be used to control the boundary layer growth.

2. In case of forced convection, the velocity being large. So, the Prandtl number (Pr) has no effective dominance over velocity and concentration fields but has significant effect on the tem

3. With the increase of magnetic parameter $(M)$ the temperature first decrease and after that start to increase, so we get a cross flow.

4. Schmidt number $\left(S_{i}\right)$ can be used very effectively to control the concentration of the flow field

5. The Eckert number $(E c)$ ) has no effective priority over velocity and concentration profiles, but it has significant increasing effect on temperature profiles.

6. In case of velocity index $(p)$, the pseudo-plastic and dilatants fluids show completely opposite behavior for temperature and concentration profiles.

7. Due to power-law fluid index $(n)$ for accelerated flow $(p=\mathbf{1 . 0})$ and decelerated flow $(p=-0.5)$, There is an opposite behavior in the concentration field. 


\section{References}

Andersson, H. I., K. H. Bech, and B. S. Dandapat. 1992. Magnetohydrodynamic flow of a power-law fluid over a stretching sheet. Int. J. Non-linear Mech. 27: 926-936.

Cess, R. D. 1966. The effect of Radiation upon the forced convection heat transfer Appl. Sci. Res. 10: 12691277.

Chen, C. H. 2008. Effects of magnetic field and suction/injection on convective heat transfer on nonNewtonian power- law fluids past a power-law stretched sheet with surface heat flux, Int. J. Thermal Sci. 47: 954-961.

Cortell, R. 2008. Effects of viscous dissipation and radiation on the thermal boundary layer over a nonlinearly stretching sheet, Physics Letters. 372: 631-636.

Dandapaat, B. S. and A. S. Gupta. 2005. Flow and heat transfer in a viscoelastic fluid over a stretching sheet, Int. J. Non-Linear Mech. 40: 215-219.

Datti, P. S., K. V. Prasad, M.S. Abel, and A. Joshi. 2005. MHD viscoelastic fluid flow over a non-isothermal stretching sheet. Int. J. Eng. Sci. 42: 935-946.

Elbashbeshy, E. M. A. 1998. Heat Transfer over a Stretching Surface with Variable Surface Heat Flux. $J$. Phys. D: Appl. Phys. 31: 1951-1954.

Erickson, L. E., L.T. Fan and V. G. Fox. 1966. Heat and mass transfer on a moving continuous moving surface. Industrial \& Engineering Chemistry Fundamentals 5: 19-25.

Glauert, M. B. 1961. A study of the magnetohydrodynamic boundary layer on a flat plate, J. Fluid Mech. 10: 276-288.

Howell, T. G., D. R. Jeng, and K. J. De Witt. 1997. Momentum and heat transfer on a continuous moving surface in a power law fluid. Int. J. of Heat and Mass Transfer 40: 1853-1861.

Mahmoud, M.A.A. and M.A.E. Mahmoud.2006.Analytical solutions of hydromagnetic Boundary-Layer flow of a non-Newtonian power-law fluid past a continuously moving surface. Acta Mechanica 181: 83-89.

Rahman, M. M. and M. A. Sattar. 2006. Magnetohydrodynamic convective flow of a micropolar fluid past a continuously moving vertical porous plate in the presence of heat generation/absorption. ASME J. Heat Transfer. 128: 142-152.

Rahman, M. A., M. A. Samad, M. M. Rahman and M. Muhebujjaman. 2008. Numerical Study of MHD Forced Convective Flow of a Micropolar Fluid Past a Non-Linear Stretching Sheet with a Variable Viscosity. Dhaka Univ. J. Sci. 57: 243-248.

Rahman, M. M., M. A. Rahman, M. A. Samad and M. S. Alam. 2009. Heat Transfer in a Micropolar Fluid Along a Non-Linear Stretching Sheet with a Temperature-Dependent Viscosity and Variable Surface Temperature. Int. J. of Thermophysics. $\mathbf{3 0 .}$

Raptis, A. 1998. Flow of a micropolar fluid past a continuously moving plate by the presence of radiation, Int. J. Heat Mass Transfer 41: 2865-2866.

Sakiadis, B.C. 1961. Boundary Layer Behaviour on Continuous Solid Surfaces: I. The boundary layer equations for two-dimensional and axisymmetric flow. AICHE J. 7: 26-28.

Vajravelu, K. and A. Hadjinicolaou. 1997. Convective Heat Transfer in an Electrically Conducting Fluid at a Stretching Surface with Uniform Free Stream. Int. J. Eng. Sci. 35: 1237-1244.

(Manuscript received on 14 May, 2014; revised on 12 March, 2015) 